\title{
Terahertz air-core microstructure fiber
}

\author{
Ja-Yu Lu, ${ }^{1}$ Chin-Ping Yu, ${ }^{1}$ Hung-Chung Chang, ${ }^{1}$ Hung-Wen Chen, ${ }^{1}$ Yu-Tai Li, ${ }^{2}$ \\ Ci-Ling Pan, ${ }^{2}$ and Chi-Kuang Sun ${ }^{1,3, a)}$ \\ ${ }^{1}$ Department of Electrical Engineering and Graduate Institute of Photonics and Optoelectronics, \\ National Taiwan University, Taipei 10617, Taiwan \\ ${ }^{2}$ Graduate Institute of Electro-Optical Engineering, National Chiao-Tung University, Hsinchu 30056, \\ Taiwan \\ ${ }^{3}$ Research Center for Applied Sciences, Academia Sinica, Taipei 10617, Taiwan
}

(Received 28 August 2007; accepted 8 January 2008; published online 14 February 2008)

\begin{abstract}
A low-loss terahertz air-core microstructure fiber is demonstrated for terahertz waveguiding. Substantially low attenuation constant less than $0.01 \mathrm{~cm}^{-1}$ has been achieved and the guiding wavelength is found to be tunable by linear scaling the fiber size. The experimental results well agree with the simulation based on the finite-difference frequency-domain method, which interprets the guiding mechanism as the antiresonant reflecting waveguiding. The simulated modal pattern shows that most terahertz field is concentrated inside the central hollow air core and is guided without outside interference, which has high potential for guiding intense terahertz waves with minimized loss. (C) 2008 American Institute of Physics. [DOI: 10.1063/1.2839576]
\end{abstract}

Terahertz waveguiding is extensively studied in recent years with mechanisms based on previous microwaves ${ }^{1-3}$ or photonics $^{4-8}$ experiences. High dielectric $\operatorname{loss}^{4-6}$ and finite metal conductivity ${ }^{1-3}$ at the terahertz range challenge the optical-fiber-type ${ }^{4-8}$ or metal-waveguide-type guiding ${ }^{1-3}$ design. Because the obstacle to realize low-loss terahertz propagation in a dielectric waveguide is the ultrahigh material absorption loss, it could be feasible to use the hollowcore fibers with a structured cladding to effectively confine the transverse modes for low loss waveguiding. In this letter, we propose a terahertz air-core microstructure fiber (TAMF) for low loss guiding of terahertz radiation. The TAMFs are composed of a central hollow core and a cladding layer formed by periodic arrangements of flexible and commercial available polytetrafluorethylene (Teflon) plastic tubes. Four TAMFs with different hollow core sizes and Teflon tube pitches were made for studying their terahertz waveguiding characteristics. Our study shows that the measured effective attenuation constants, including coupling loss and propagation loss, could be much less than $0.01 \mathrm{~cm}^{-1}$ at specific terahertz transmission bands and the frequency of the hightransmission terahertz bands could be adjusted by linear scaling the fiber size. Simulation based on a finite-difference frequency-domain (FDFD) method ${ }^{9}$ shows good agreements with the experimental results, suggesting a guiding mechanism similar to that of the antiresonant reflecting optical waveguiding (ARROW). ${ }^{10,11}$ The simulated modal patterns at the terahertz transmission bands are with a centrally localized core mode, revealing that most terahertz field is well confined inside the central hollow air core and is guided with minimized outside interference.

To design a TAMF for effective delivery of terahertz radiation, a FDFD method ${ }^{9,12}$ was employed for the analysis of the air-core waveguide structures, of which the cross sections are shown in the insets of Fig. 1(a) and 1(c). As shown in the inset of Fig. 1(a), two simulated TAMF are assumed to be composed of Teflon tubes with the outer and inner diameters $d_{o}$ and $d_{i}$ being 2.08 and $1.68 \mathrm{~mm}$, respectively. These

\footnotetext{
a) Author to whom correspondence should be addressed. Electronic mail: sun@cc.ee.ntu.edu.tw.
}

Teflon tubes were assumed bound together in a triangular lattice with the distance between the centers of two adjacent tubes (i.e., pitch) $\Lambda=2.08 \mathrm{~mm}$. Without the center seven Teflon tubes, one can have a TAMF with one-ring or two-ring Teflon tubes left as the cladding region, respectively. The central air-core regions for both simulated structures are with an effective diameter of $2.64 \Lambda=5.5 \mathrm{~mm}$. Figures 1 (a) and 1(b) show the simulated modal refractive indices and the corresponding propagation loss of the guided modes on both TAMF waveguides as a function of frequency. The modal effective index of the guided mode is defined as real part $\beta$ of the modal propagation constant divided by the free-space wavenumber $k_{0}$. Just like in the standard microwave metallic waveguide filled with air for which $\operatorname{Re}(\beta)<k_{0}$, the guided mode in the TAMF waveguide also obeys $\operatorname{Re}(\beta)<k_{0}$ and, thus, has an effective index smaller than 1 . This is also true for the air-core photonic bandgap fiber. ${ }^{13}$ The propagation loss is obtained from the calculated imaginary part $\beta$ of the modal propagation constant. Centrally confined guided modes can be solved over the frequency range between 0.3 and $1 \mathrm{THz}$, except for the frequencies near $700 \mathrm{GHz}$. In Fig. 1(a), we can observe that as the frequency approaches the discontinuities in the modal dispersion curves, relatively large propagation loss at such frequencies can be found in Fig. 1(b). Both one-ring and three-ring TAMF waveguides show similar frequency-dependent behaviors, revealing that the guiding mechanism could be just like the ARROW ${ }^{10,11}$ mode in a microstructured optical fiber. It also indicates that the characteristic of the proposed TAMF is dominated by the first cladding layer and the increase of the ring number has little influence on the modal properties of the waveguides. Detailed FDFD simulation ${ }^{9}$ indicated that these discontinuities correspond to various resonant frequencies of the cladding structure, at which the field in the air core cannot be antiresonantly reflected by the cladding in an efficient manner and, thus, leaks into the Teflon tubes, significantly increasing the propagation loss. The resonant frequencies of the cladding are determined by the various geometries of Teflon tubes, including their inner/outer diameters and shapes, as well as the index contrast of the composed materials. ${ }^{10,11}$ At other frequencies, the field is reflected by 

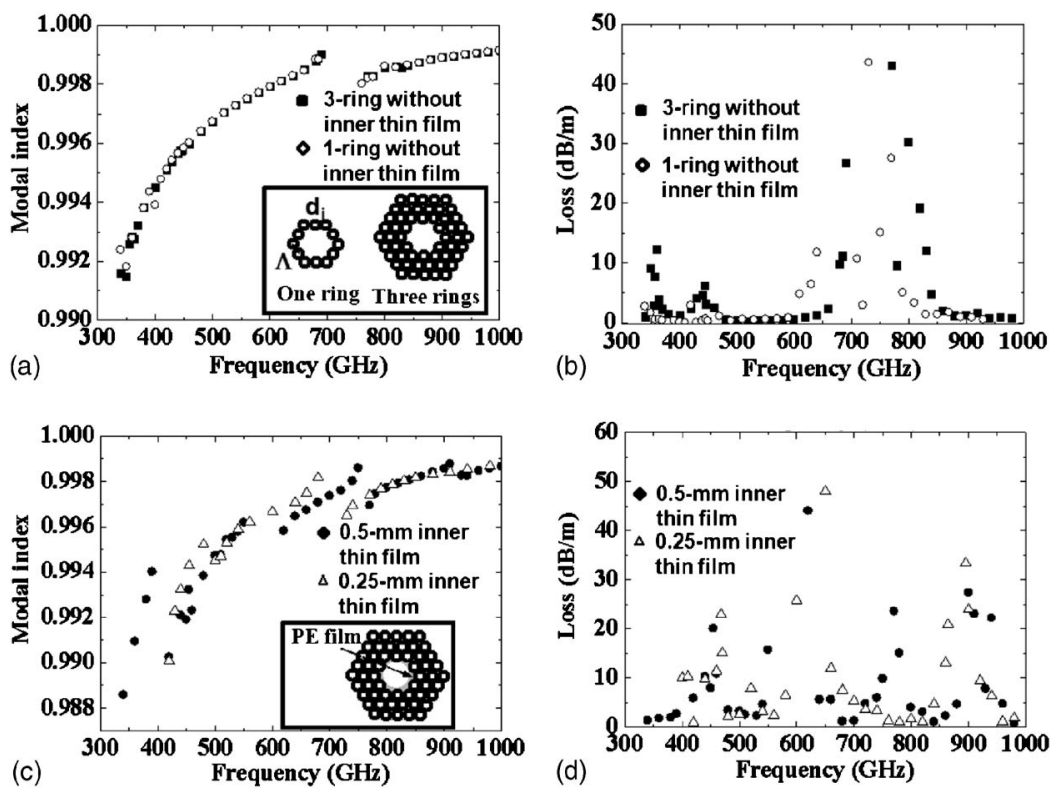

FIG. 1. (a) Simulated modal indices and (b) simulated propagation losses of the guided modes in the air-core waveguides with the one-ring and three-ring Teflon tube structures. (c) Simulated modal indices and (d) simulated propagation losses of the guided modes on a three-ring air-core waveguide with a PE inner film with different film thicknesses. Insets show the schematic drawings of the simulated structure cross sections. the cladding to support a centrally localized guided mode with a much lower propagation loss. In practical practice to realize such a waveguide, we attached a thin polyethylene (PE) film on the side wall of the air core for structure stability [inset of Fig. 1(c)]. Figure 1(c) shows the simulated modal dispersion curves for a three-ring air-core terahertz waveguide with this additional inner PE film with a thickness of 0.50 or $0.25 \mathrm{~mm}$. Comparing Fig. 1(c) with 1(a), one can observe that more discontinuities appear, corresponding to extraresonant frequencies induced by the insertion of this thin PE film. In Fig. 1(d), the relatively high propagation loss also reveal the same phenomenon due to additional resonant frequencies [compared to Fig. 1(b)] induced by the PE film. In addition, a thicker inner film will lower the resonant frequency, induce more propagation loss peaks, and make the transmission bands narrower [shown in Fig. 1(d)]. This simulated result indicates that the geometries of the Teflon tubes and the inner thin film need to be carefully designed for the desired propagation characteristics.

In experiment, we handmade four TAMFs without any furnace and fiber drawing processes, labeled as MF1 to MF4 which are with an inner/outer diameters of $1.68 / 2.08$, $1.19 / 1.49,0.96 / 1.26$, and $0.81 / 1.11$, respectively. They were packed in the hexagonal closest arrangement with different inner and outer tube diameters. We first stacked the central seven plastic tubes with a PE heat-shrinkable tubing for fastening, then added the cladding tubes. The bundled tubes with a hexagonal arrangement were fastened with another PE heat-shrinkable tubing. Finally, the central seven plastic tubes were removed to form a hollow core with the inner PE film left [as shown in Figs. 2(c) and 2(d)]. The fabricated TAMFs were with a similar structure to that shown in Fig. 1(c) but with a five-layer cladding and a length of $20 \mathrm{~cm}$. We adopted a Martin-Puplett polarizing interferometer and broadband terahertz pulses to measure the terahertz transmission spectra after these were guided through the TAMFs. To block the terahertz transmission through the cladding region, aluminum foils were attached to cover the cladding region at the fiber output end. Figures 2(a) and 2(b) show the measured normalized transmission spectra of the MF1 and MF2 fibers with multiple transmission bands and two strong water vapor absorption lines (at 560 and Downloaded 16 Feb 2009 to 140.112 .113 .225 . Redistribution subje
$762 \mathrm{GHz}$ ). Figures 2(c) and 2(d) show the photos of the finished MF1 and MF2 fibers and the calculated modal patterns of the transmission peaks in each MF. We used the FDFD method $^{12}$ to calculate the cladding structure's photonic bandgap spectrum. From the calculated bandedge diagrams, ${ }^{12}$ we found that there are four photonic bandgaps (not shown here) for the MF1 fiber with narrow bandwidths below $1 \mathrm{THz}$, of which the frequency and bandwidth do not agree well with the experimental results, indicating that the photonic bandgap effect does not dominate the observed guiding mechanism. On the other hand, the measured terahertz transmission spectra [solid lines in Figs. 2(a) and 2(b)] match well with our simulated results by the FDFD method [black dashed lines in Figs. 2(a) and 2(b)]. Two dominant terahertz transmission bands peaked at 490 and $637 \mathrm{GHz}$ were found for MF1 and we attribute them to the ARROW-like modes resulting from the antiresonance of the Teflon tubes, based on the FDFD simulation. The FDFD calculated modal refractive indices of both waveguides are also shown in Figs. 2(a) and 2(b) as red dashed lines with multiple discontinuities, of which the frequencies coincide well with the measured spectral transmission gaps, which again suggests the ARROW-like ${ }^{10,11}$ waveguiding mechanism. From the simulated modal patterns of the transmission peaks shown in Figs. 2(c) and 2(d), one could find that most terahertz power is well confined in the central hollow core and is transmitted in the low-loss air.

From the measured terahertz transmission spectra, we can observe only one major transmission band in MF2, MF3, and MF4, while the measured transmission band shifts to a higher frequency as the pitch size becomes smaller. Figure 3 shows the relation between the measured central guiding wavelength and the central hollow core size. A linear dependency can be found, which reveals that the major transmission band could be adjusted by linearly scaling the TAMF size. The measured effective attenuation constant, by comparing the terahertz power before and after the fiber without calibrating the air loss and the coupling loss, can be found to be as low as $0.002 \mathrm{~cm}^{-1}$ at the $770 \mathrm{GHz}$ transmission peak of MF1. Since the measured attenuation constant includes the coupling loss, our study also suggests a coupling efficiency higher than $50 \%$ in the TAMF. The theoretical attenuation to AIP license or copyright; see http://apl.aip.org/apl/copyright.jsp 

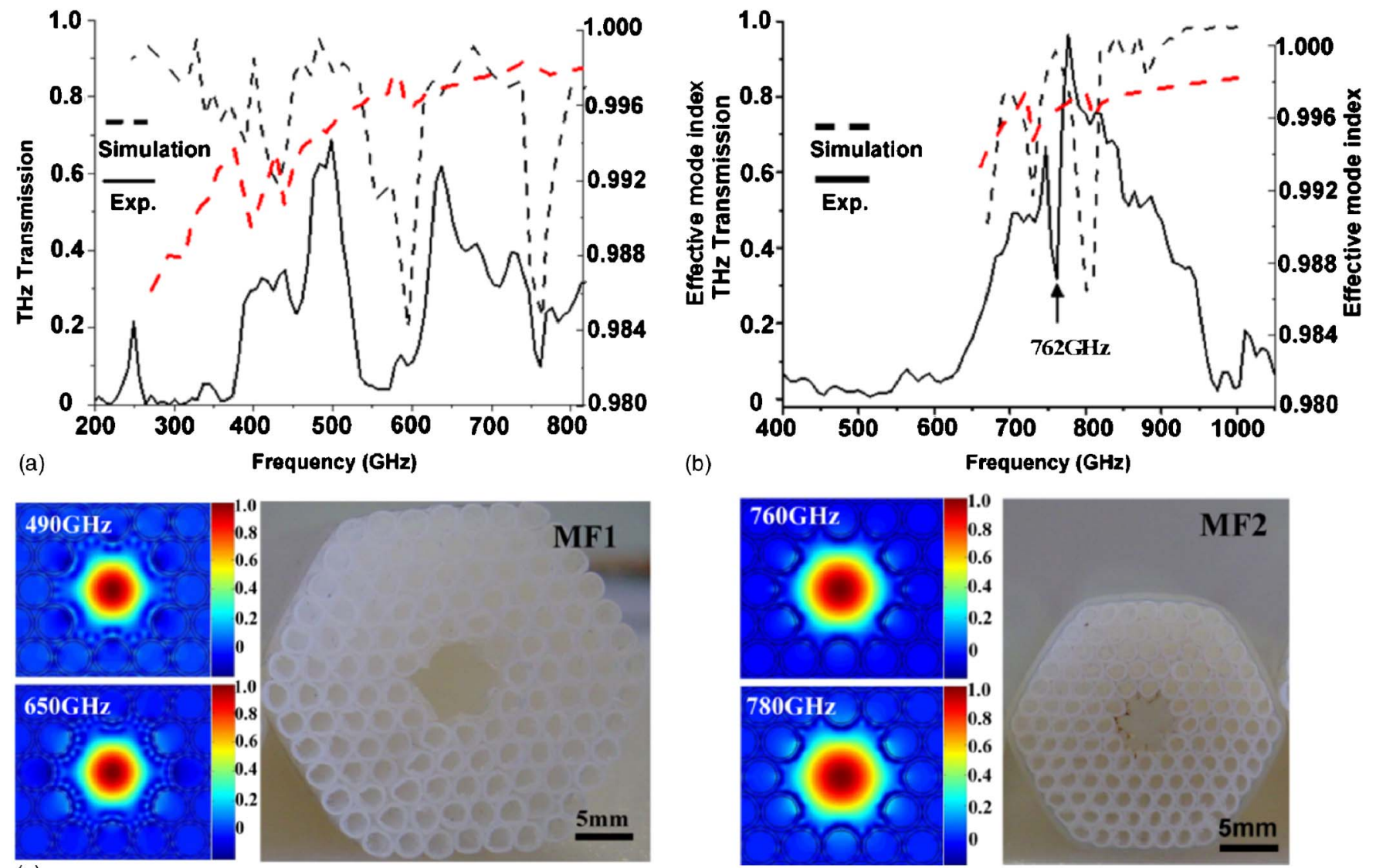

(c)

(d)

FIG. 2. (Color online) The measured (solid line) and simulated (black dashed line) transmission spectra of the (a) MF1 fiber and the (b) MF2 fiber. (c) and (d) show the FDFD simulated modal patterns at transmission peak wavelengths and photos of the fabricated MF1 and MF2 fibers. Red dashed lines represent the simulated effective modal refractive index.

constant at this transmission peak is $5 \times 10^{-4} \mathrm{~cm}^{-1}$.

In conclusion, we have demonstrated a low-loss terahertz air-core microstructure fiber. Substantially low attenuation constant less than $0.002 \mathrm{~cm}^{-1}$ has been achieved, and the guiding wavelength is also found to be adjustable by linearly scaling the fiber size. The experimental results well agree with the simulation based on a finite-difference frequency-domain method, which interprets the guiding mechanism as the antiresonant reflecting waveguiding. The simulated modal pattern shows that most terahertz field is

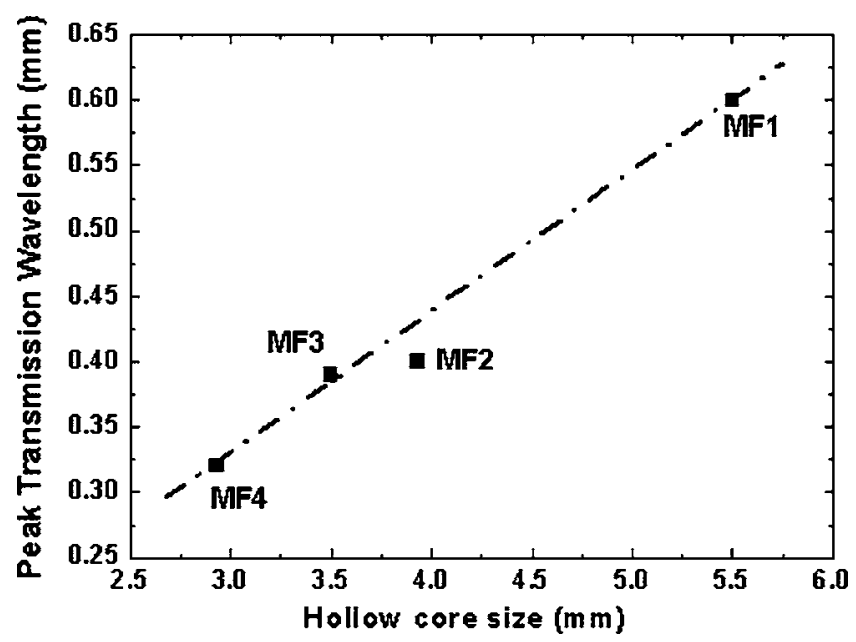

FIG. 3. Relation between the central guiding wavelength and the hollow core size. The dotted line is a linear fit. concentrated inside the central hollow air core and is guided without outside interference, with a high potential for guiding intense terahertz waves with minimized loss.

This work was sponsored by the National Science Council of Taiwan under Grant Nos. 96-2628-E-002-043-MY3 and 96-2120-M-002-014, the Aim for the Top University program of the Ministry of Education, Taiwan, the Program for Promoting Academic Excellence of University Project (Phase II) in National Chiao Tung University, and the NTU Center for Medical Excellence.

${ }^{1}$ J. Harrington, R. George, P. Pedersen, and E. Mueller, Opt. Express 21, 5263 (2004).

${ }^{2}$ K. Wang and D. M. Mittleman, Nature (London) 432, 376 (2004).

${ }^{3}$ R. Mendis and D. Grischkowsky, Opt. Lett. 26, 846 (2001).

${ }^{4}$ R. Mendis and D. Grischkowsky, J. Appl. Phys. 88, 4449 (2000).

${ }^{5}$ H. Han, H. Park, M. Cho, and J. Kim, Appl. Phys. Lett. 80, 2634 (2002).

${ }^{6}$ M. Skorobogatiy and A. Dupuis, Appl. Phys. Lett. 90, 113514 (2007).

${ }^{7}$ L.-J. Chen, H.-W. Chen, T.-F. Kao, J.-Y. Lu, and C.-K. Sun, Opt. Lett. 31, 308 (2006).

${ }^{8}$ H.-W. Chen, Y.-T. Li, J.-L. Kuo, J.-Y. Lu, L.-J. Chen, C.-L. Pan, and C.-K. Sun, Opt. Lett. 32, 1017 (2007).

${ }^{9}$ C. P. Yu and H. C. Chang, Opt. Express 12, 6165 (2004).

${ }^{10}$ T. P. White, R. C. McPhedran, C. M. Sterke, N. M. Litchinitser, and B. J. Eggleton, Opt. Lett. 27, 1977 (2002).

${ }^{11}$ N. M. Litchinitser, A. K. Abeeluck, C. Headley, and B. J. Eggleton, Opt. Lett. 27, 1592 (2002).

${ }^{12}$ C. P. Yu and H. C. Chang, in Electromagnetic Theory and Applications for Photonic Crystals, edited by K. Yasumoto (Marcel Dekker/CRC, Boca Raton, Florida, 2006), Chap. 7, pp. 351-400.

${ }^{13}$ K. Saitoh and M. Koshiba, Opt. Express 11, 3100 (2003). 